\title{
DIE BEDEUTUNG DER IDENTITÄT BEIM VERLUST ODER ERHALT DES SPANISCHEN UNTER DEN JUNGEN EMIGRANTEN DER ZWEITEN GENERATION IN DEUTSCHLAND.
}

D ie Untersuchungen von Giles und Johnson (1981), Giles und Byrne (1982) und Gardner (1982 und 1985), unter anderen, haben gezeigt, daß der ethnolinguistische Identitätstyp den Fremdsprachenlernproze $\beta$ in starkem Maße beeinflußt, d.h. die Art der Zweisprachigkeit, die der Lernende erlangt. $\mathrm{Ob}$ die Bilingualität als bereichernd oder aber als beeinträchtigend erlebt wird, hängt im wesentlichen von der sozialen Akzeptanz der implizierten ethnolinguistischen Gruppen ab.

Von dieser Grundlage ausgehend stellen wir die Hypothese auf, daß der ethnolinguistische Identitätstyp nicht nur den Lemprozeß einer Fremdsprache, sondern auch den Verlust einer einst beherrschten Sprache beeinflußt, wenn nicht sogar wesentlich bestimmt. Die Gründe, die den Erwerb einer Fremd sprache behindern, könnten die gleichen sein wie die, die den Verlust einer Sprache bewirken und beschleunigen: der Mangel an integrativer Motiva- tion bzgl. der Herkunftsgruppe in Verbindung mit einem starken Zugehörigkeitsgefühl zur dominanten ethnolinguistischen Gruppe.
Das heißt jedoch nicht, daß eine starke ethnolinguistische Identität mit der Minoritätengruppe ein ebenfalls intensives Zugehörigkeitsgefühl mit der dominierenden Gruppe unmöglich macht. Wenn dieses Identitätsbewußtsein von einer integrativen Motivation im Sprachgebrauch getragen wird, ist es durchaus möglich, daß das Individuum eine doppelte ethnolinguistische Identität entwickelt.

Anhand mehrerer theoretischer Modelle wurde der Versuch unternommen, die unterschiedlichen möglichen Reaktionen des Individuums auf die meist konfliktreichen bikulturellen und bilingualen Situationen zu klassifizieren. Child (1967), Tosi (1977) und Siguán (1976) unterscheiden drei verschiedene Reaktionsarten der jugendlichen Emigranten, wobei sie bei den ersten beiden übereinstimmen: 
1) Abwendung von der Herkunftsgruppe und Versuch der Assimilation an die dominante Gruppe;

2) Rückzug in die Herkunftsgruppe; diese Reaktion hat, wie Tosi und Siguán betonen, die Annahme der Diglossie und den daraus folgenden mehr oder weniger starken Verlust der Muttersprache zur Folge.

3) Bei der dritten möglichen Reaktion sprechen Child und Siguán von einer Haltung der Ambiguität und Apathie. Offensichtlich handelt es sich hierbei um die unglücklichste Lösung, denn sie versagt dem Individuum die Entwicklung einer gesunden Identität (sei es mit der Minoritätengruppe, mit der dominanten Gruppe oder mit beiden Gruppen gleichzeitig). Wir sind der Meinung, daß die Ablehnung von "allem" in vielen Fällen unbewußt ist und auf der Tatsache beruht, daß das Individuum sich nirgends zuhause fühlt oder aber auch, weil eine Entscheidung eine gewisse Anstrengung verlangt.

Nur Tosi beschreibt die Reaktion der Auflehnung (rebel reaction), welche dem Konflikt zwischen den Werten beider Gruppen nicht ausweicht, sondern inn regelrecht sucht, um inn bewußt auszutragen. Dieses Verhalten ermöglicht die Entwicklung einer doppelten, ausgewogenen und bereichernden Identität.

Unserer Erfahrung nach überwiegt die dritte von Child und Siguán beschriebene Reaktion, aber es gibt auch einige wenige Individuen, die der von Tosi beschriebenen Reaktion der Auflehnung zugeordnet werden können. Aus diesem Grunde schlagen wir ein Beschreibungsmodell vor, welches alle vier Reak- tionsmöglichkeiten umfaßt, demnach also eine Synthese der drei genannten Klassifikationsmodelle darstellt. Unser Modell kann natürlich nur orientativen Charakter haben, denn das menschliche Verhalten ist zu kompliziert, um jederzeit problemlos in ein Schema eingeordnet werden zu können. In vielen Fällen treffen wir sogar auf vollkommen widersprüchliche Verhaltensweisen und Einstellungen, was durchaus normal und häufig sogar positiv zu bewerten ist, da es ein Zeichen dafür sein kann, daß das betreffende Individuum verschiedene Möglichkeiten abwägt, um die für es jeweils beste zu wählen.

In einer soziolinguistischen Studie über den Sprachverlust des Spanischen unter den Emigranten der zweiten Generation in Deutschland, welche wir im Jahre 1988 in Form eines je einstündigen Interviews ${ }^{2}$ bei 27 spanischen Jugendlichen durchgeführt haben ${ }^{3}$, versuchten wir, unsere Hypothese mit Hilfe des oben beschriebenen Klassifikationsmodells zu überprüfen, d.h. zuerst teilten wir unsere Informanten einer der vier Reaktionsgruppen zu und analysierten anschließend die Spanischkenntnisse der einzelnen Gruppen als Ganzes". Dabei gelangten wir zu folgenden Ergebnissen:

\section{Versuch der Assimilation} an die dominante Gruppe:

Unter unseren Informanten haben wir nur einen Extremfall in dieser Richtung gefunden, welcher zum fast völligen Verlust der spanischen Sprache und Kultur geführt hat. Es handelt sich um Mónica (11). Obwohl ihre Eltern sehr schlecht deutsch sprechen, wendet sie sich nur in dieser Sprache an sie, weil sie Spanisch zwar verstehe aber nicht spreche. Wir haben hier einen Fall von völliger Ablehnung der 
spanischen Sprache und Kultur, welche die Einsprachigkeit oder im besten Falle eine sehr begrenzte rezeptive Bilingualität zur Folge hat. Die Eltern sehen diesen Verlust nicht als negativ, im Gegenteil, sie ziehen es vor, daß ihre Tochter ganz und gar in die deutsche Gesellschaft integriert ist, wovon sie sich einen sozioökonomischen Aufstieg für diese versprechen. Ihrer Meinung nach könnte der Erwerb des Spanischen auf diesem Wege hinderlich sein. Diese radikale Reaktion mag auch teilweise durch das völlige Fehlen jeglicher institutioneller Unterstützung für die spanische Sprache und Kultur am Wohnort der Informantin (Brühl bei Köln) bedingt sein. Es gibt weder eine spanische Schule, noch ein spani-sches Kulturzentrum oder eine Pfarrei. Zum Zeitpunkt der Untersuchung war es nicht einmal möglich, eine spanische Zeitung oder Zeitschrift in der Stadt zu kaufen. Auch der Anteil der spanischen Bevölkerung und damit die Möglichkeit des Kontaktes zu anderen Spaniern ist sehr gering.

Außer Mónica haben noch weitere neun Jugendliche beschlossen, sich ganz der deutschen Gesellschaft anzu-schließen (6 Mädchen und 3 Jungen) und ihren spanischen Teil als rein anekdotisch, aber nicht als entscheidend anzusehen. Bei ihrer Identitätsbildung spielt die spanische Komponente eine verschwindend kleine Rolle, welche sie nicht selten lieber vergessen würden. So sagt z.B. Carolina (10): - soy alemana + pero mis padres son españoles

Alexandra (13) sagt, daß sie sich wie eine Deutsche fühle. Sie möchte nicht nach Spanien zurück und sagt sogar, daß Spanien ihr "egal" sei.

Itziar (13) erählt: - no me se ve (sic) mucho que soy española + sólo por el nombre que no me gusta nada pero + si no no - ¿te gustaría más ser alemana? - medio alemana medio española me gustaría tú te sientes como uno más aqui ¿no? - como un alemán + sí - ¿te molesta que los amigos de España te digan alemana? - no me importa mucho + si les dices que soy española no dicen nada y siempre dicen alemana + no les puedes decir que eres española porque no te lo creen + dicen + has nacido en Alemania eres alemana.

Sie wird eher von der deutschen als von der spanischen Gruppe akzeptiert, denn, wie sie selbst sagt, unterscheidet nur ihr markanter Name sie von den Deutschen. Im Gegensatz dazu sieht die spanische Gemeinschaft sie nicht als vollwertiges Mitglied an, wahrscheinlich nicht nur, wie sie glaubt, weil sie in Deutschland geboren wurde, sondern auch, weil sie sich sprachlich von ihnen unterscheidet

Carmen (16) erzählt über ihre Erfahrung in Spanien: - dicen "Heil Hitler" como Hitler ino? algo asi - iy eso te molesta? - como no me encuentro asi alemán alemana de verdad + pues no + no me molesta y además + como yo no he vivido en esos tiempos yo no tengo la culpa de eso + de nada + por eso no me molesta pero me + estoy aqui y me dicen española + estoy en España y me dicen alemana pues + ya ni sabes de lo que eres al final - ¿pero tú, qué crees que eres? ¿cómo te ves tú más, como alemana o como española? - yo + me parece que pienso más como una alemana de ... igual de salir a la calle hasta tarde + ir a la discoteca + y mi madre + pues claro + ella piensa como una española y me tiene más atada + pero yo me parece que pienso más como una alemana.

Nicht nur die Tatsache, daß sie ausdrücklich sagt, daß sie sich eher als Deutsche denn als Spanierin fühle, sondern auch ihre Reaktion auf die Anspielung auf die Hitlerzeit von Seiten der Spanier läßt darauf schließen, daß sie sich ganz der deutschen Gruppe zugehörig fühlt, denn wenn sie sich als Spanierin fühlen würde, hätte sie nicht zu erwähnen brauchen, daß sie zu jener Zeit noch nicht geboren war.

Bei einer anderen Gelegenheit ließ der Gebrauch der ersten Person statt der dritten darauf schließen, daß Carmen sich als Mitglied der deut-schen Gesellschaft und nicht der Gruppe der Ausländer sieht: - 
aqui habla la gente muy mal de los turcos + que esto + que han venido aqui a quitarnos trabajo y todo eso.

Ester (19): - tú te sientes completamente alemana ¿no? - si estoy aqui si + si estoy en España + también (...) si + porque es que yo he nacido aquí.

Agustín (14): - ¿piensas volver? - ¿volver? - volver a España - si yo no he estado nunca en España - pero tú eres español ¿no? - bueno + no sé + como he estado toda mi vida aqui + me parece que no.

Agustín definiert sich eindeutig als Deutscher, und er findet es sogar absurd, von einer "Rückkehr" zu sprechen. Ein spanischer Ursprung, zu dem er zurückkehren könnte, ist ihm nicht mehr bewußt. Er sieht seine Wurzeln alleine in Deutschland.

Jesús (29): - ¿te sientes marginado? ¿te sientes como un extraño en Alemania? - muchas veces si. ¿por qué? - porque tengo el pasaporte español y si voy a un secretareado (sic) y ven que soy español ya me miran con otros ojos + pero después cuando empiezo a hablarles el alemán - asi que en realidad ni eres español ni alemán porque - si muchas veces si - me siento más alemán que español.

Jesús hat sich für die Zugehörigkeit zur deutschen Gruppe entschieden. Allerdings läßt diese ihn bei Gelegenheit spüren, daß er kein vollwertiges Mitglied ist, wenn auch nur auf amtlicher Ebene.

Diaz (30): - no me atrae ya España como a los demás + la última vez que fui (vor 11 Jahren) fue un poco decepcionante fui con cuatro semanas y a las dos semanas me vine + no tienes amigos (...) la manera de expresarte + el idioma también lo hace + no (daß man keine

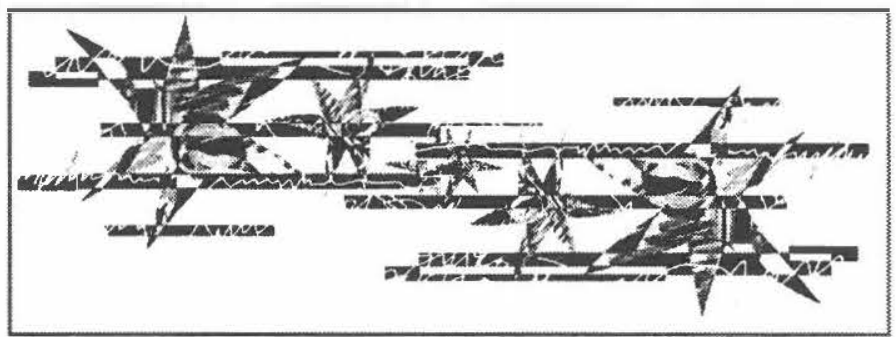

Freunde in Spanien hat) me siento español + pero no me atrae ir alli (...) cuando me encontraba a turistas alemanes en España me gustaba estar con ellos (Nach wenigen Tagen fing er an, sich in Spanien unwohl zu fühlen.) (...) ya te sientes extranjero (...) yo me sentia más extranjero en España que aqui(...) wir leben in einem Land in dem wir nicht geboren sind und wir müssen damit leben (...) ich habe mich von Anfang an mehr auf die deutsche Seite geschlagen.

Obwohl er sagt, daß er sich als Spanier fühle und trotz seiner intensiven Mitarbeit im spanischen Club (er ist Vorstandsmitglied), deuten alle seine anderen Antworten und seine ganze Einstellung auf das Gegenteil hin. Vielleicht besteht er auf seiner spanischen Identität, weil sein dunkelhäutiger Typ ihm eine völlige Integration in die deutsche Gesellschaft unmöglich macht: - si eres extranjero y estás sólo y estás junto con alemanes y hablas el alemán bien + que ellos apenas lo notan + como me lo dicen muchas veces + entonces ya te tratan de otra manera ¿no? pero si estás un grupillo de españoles o extranjeros junto entonces si + ya notas de otra manera + ya es diferente + ya no es lo mismo + según también con quién trates + qué personas sean + si son gente de la calle lo notas más + si es en el trabajo lo notas menos + quizás debido al puesto que yo tengo de trabajo ino? yo con los alemanes con los maestros me entiendo + me tratan de otra manera porque soy encargado + ya es otra cosa + eres extranjero pero ya eres algo más.

Auf die Frage, ob er sich ausgeschlossen fühlt, antwortet er: - es lógico + te lo dan a entender.

Dann wiederum sagt er: - me entiendo mejor con alemanes que con españoles + los alemanes los entiendo + los comprendo antes + o sea + sé por donde van + un español creo que tiene muchas caras.

Er hat die Ablehnung von Seiten beider Gemeinschaften erlebt und wird sich auch weiterhin damit abfinden müssen. Jedoch ist er nicht bereit, diesen Konflikt bewußt auszutragen: - los problemas que tienen los jóvenes emigrantes españoles se los buscan ellos + que nos tratan mal y que somos extranjeros + se exagera mucho.

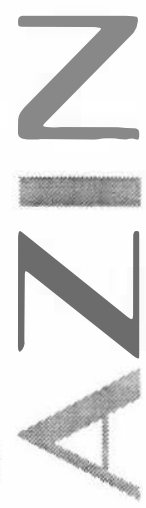


Diese Einstellung macht es natürlich unmöglich, daß er seinen Konflikt, der ja offensichtlich existiert, auf intellektueller Ebene austrägt. Er befindet sich gewissermaßen im "Niemandsland", denn er gehört keiner von beiden Gruppen voll an. Weder die Spanier noch die Deutschen akzeptieren ihn als einen der ihrigen. Es handelt sich um einen Fall von völliger Entwurzelung. Trotzdem hat Diaz die Entscheidung gefaßt, sich mehr der deutschen Gruppe anzuschließen, auch wenn das schwierig ist und obwohl er nie als Deutscher gelten können wird. Er beschreibt seine Situation so: - siempre se es extranjero + extranjeros somos todos en todas partes.

Sicher waren es die finanziellen Vorteile, die die deutsche Gesellschaft ihm bietet, die ihn in diese Richtung gewiesen haben. Ein klares Zeichen dafür ist die Tatsache, daß seine Tochter kaum Spanisch spricht, obwohl auch seine Frau eine spanische Emigrantentochter ist, und obwohl die spanische Sprache und Kultur an ihrem Wohnort eine breite institutionelle Unterstützung finden. - con la nin̄a hablo en un $90 \%$ en alemán porque quiero que el alemán lo practique conmigo.

Er will seine Tochter nicht in die spanische Schule schicken, obwohl er glaubt, daß es eigentlich seine Pflicht ist. Er fürchtet, sie

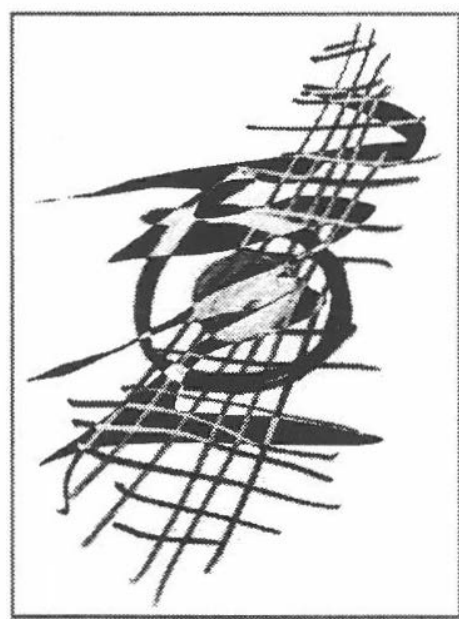
durch den doppelten Unterricht eventuell zu überlasten, und in diesem Falle hält er es für wichtiger, daß sie Deutsch lernt als Spanisch.

Keines der Mitglieder der ersten Gruppe möchte zurück nach Spanien, und alle versichern sie, kein "Heimweh" nach Spanien zu haben. Auf die Frage, was sie machen würden, wenn sie eine Million DM hätten, antwortete keiner dieser Gruppe, daß er nach Spanien zurückgehen würde. Diese Frage hatten wir hauptsächlich mit der Absicht gestellt herauszufinden, ob unsere Informanten lediglich aus finanziellen Gründen in Deutschland bleiben, was man mit Sicherheit von der Generation ihrer Eltern annehmen kann. Fast alle Emigranten der ersten
Generation, mit denen wir gesprochen haben, versicherten uns, daß sie sofort nach Spanien zurückkeh. ren würden, wenn sie nicht aus finanziellen Gründen gezwungen wären, in Deutschland zu bleiben.

\section{Rückzug in die Herkunfts-gruppe:}

Die zweite Gruppe besteht aus fünf Jugendlichen (drei Jungen und zwei Mädchen). Wir sprechen hier von "Rückzug in die Herkunftsgruppe", weil es die zweite Kategorie unseres Beschreibungsmodells ist. Man kann jedoch bei keinem dieser jungen Leute von einem wirklichen Rückzug sprechen, sondern eher von der freien Entscheidung, sich selbst eher als Spanier denn als Deutscher zu definieren, obwohl keiner von innen bestreitet, zumindest teilweise von der deutschen Kultur mitgeprägt worden zu sein. Von einer Ausnahme abgesehen, lehnen sie die deutsche Kul. tur nicht $a b$, und sie fühlen sich vollständig in sie integriert. Deshalb wäre es in unserem Falle vie. lleicht angebrachter, von einem "Verweilen in der Herkunftsgruppe" zu sprechen.

Drei der zu dieser Gruppe gehörenden Informanten haben Heimweh nach Spanien, und alle würden sie gerne zurückgehen, wenn die Arbeitsbedingungen und sozialen Umstände in Spanien das zuließen Sie alle erzählen, irgendwann einmal Probleme mit den Deutschen gehabt zu haben, weil sie Ausländer sind. In der ersten Gruppe haben nur vier von neun Informanten diese Erfahrung gemacht.

Marco Antonio (14) möchte nicht die deutsche Nationalität annehmen: - porque me gusta más ser español + porque luego soy medio alemán y medio español y no + es mejor ser de un sitio entero que medio. Er ist davon überzeugt, daß - alli en España estaría mejor. Er erzählt, daß er häufig mit seinen Eltern zum Essen ausgeht, aber daß sie noch nie in einem deutschen Restaurant waren: - no hemos entrado en ninguno. Er erinnert sich an Reibereien mit seinen Klassenkameraden: - esos los de mi clase eran muy alemanes + que no saben tener como un 
amigo a un griego o alguno de esos + hay que pelearse + como uno de mi clase del segundo que era un español (...) ese tenia todos los días una pelea con alguno de estos de mi clase + yo no he tenido ninguna pelea porque no me gustaba pelearme + nos dicen de vez en cuando "mierda de extranjero" o alguna cosa así.

Er ist davon überzeugt, daß seine Eltern eine Spanierin als zukünftige Schwiegertochter vorziehen würden.

Oberflächlich betrachtet könnte es so aussehen, als ob Marco Antonios Einstellung gegen die Deutschen in der Haltung seiner Eltern begründet sei. Aber in diesem konkreten Falle ist eine solche Erklärung sehr unwahrscheinlich, da seine jüngere Schwester, Carolina, sich ganz klar als Deutsche definiert und bei ihr keinerlei Hinweise auf eine negative Einstellung gegen die Deutschen festzustellen ist. Wir sind vielmehr der Meinung, daß es in diesem Fall sehr viel wahrscheinlicher ist, daß die Einstellung des Jungen das Resultat sehr persönlicher und individueller Erfahrungen ist, womit wir jedoch den Einfluß der Eltern in der Haltung ihrer Kinder in keiner Weise in Frage stellen möchten.

Drei weitere Informanten, die dieser Gruppe angehören, berichten davon, daß sie sich irgendwann in der Vergangenheit ausgeschlossen gefühlt haben. Sie empfinden eine klar definierte Grenze zwischen der deutschen und der spanischen Gesellschaft, jedoch sehen sie diese nicht als unüberwindbar an. Wir verweisen in diesem Zusammenhang auf die Kategorie "perceived hardness of group limits" der "social identity theory" von Tajfel and Turner.) Die Antworten unserer Informanten bzgl. dieser Frage erinnern an das Phänomen der von Franceschini beschriebenen "myth culture", in der die Herkunftskultur blind verherrlicht wird: tienen (las mujeres españolas) mejor carácter que estas alemanas + son más simpáticas - las casas españolas por dentro tienen más alegría que estas alemanas (interessant ist auch der pejorative Gebrauch des Demonstrativpronomens) - los alemanes son muy serios y no caen bien.

Susana (21): - las viviendas españolas son más calientes claras y bonitas - los alemanes piensan más en el dinero (...) los alemanes odian más a sus padres. Auf der anderen Seite gebraucht, die gieiche Informantin die erste Person, um einen deutschen Usus zu beschreiben: - decimos "novio" cuando ya estamos prometidos.

Ana Alicia (21) definiert sich als Spanierin, ohne sich jedoch der deutschen Gesellschaft zu verschließen: - los españoles son de otra forma + se animan más y los alemanes + no sé + depende.

Salvador (25): - es fácil tener amigos españoles + la forma de ser + cómo viven + el comportamiento + los alemanes tienen otra forma de vida otra forma de ser. Als wir ihn fragten, ob er denn gar nichts Deutsches an sich habe, antwortete er zweifelnd: - a lo mejor un poquito + pero + ich weiß nicht. Salvador weiß, daß seine äußere mediterrane Erscheinung eine völlige Integration in die deutsche Gesellschaft unmöglich macht: - por mi apariencia + por ejemplo + con bigote + moreno + español + ha habido un + me han hablado diferentemente como si no entendiera alemán. Auf die Frage, ob er sich ausgeschlossen fühle, antwortet er: - no me lo planteo + ich geh da vorbei an solchen Sachen. Offensichtlich weicht er einem Konflikt bewußt aus, weil es ein scmerzlicher Prozeß für ihn sein könnte. Er erzählt, daß er nach Spanien zurückgegangen war, um dort zu bleiben, aber daß es ihm unmöglich war, sich an den spanischen Lebensstil anzu passen. Aus diesem Grunde kam er wieder nach Deutschland zurück. Die Dramatik seiner Situation ist evident. $\mathrm{Er}$ sucht die Zugehörigkeit zu einer der beiden Gemeinschaften, denn diese Bindung würde inm ein Gefühl der Sicherheit verleihen. Dies bleibt inm jedoch verwehrt,

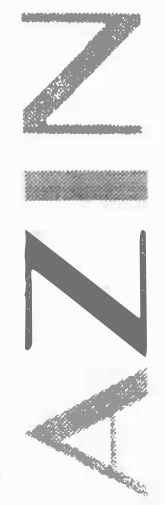


einerseits wegen seines Äußeren und andererseits wegen seiner Unfähigkeit, sich nach so vielen Jahren in Deutschland der spanischen Lebensart anzupassen. Salvador steht mit dieser Erfahrung nicht alleine da. Viele junge Emigranten der zweiten Generation versuchen aus Identitätsgründen, in Spanien Fuß zu fassen, entscheiden sich aber angesichts ihrer unsicheren Lage in diesem Land dafür, wieder nach Deutschland zurückzukehren. Salvador spricht stellvertretend für sie alle, wenn er die Bedingungen aufzählt, die erfültt werden müßten, damit diese jungen Leute wieder nach Spanien zurückkehren könnten: - un puesto de trabajo fijo y seguridad social (im weitesten Sinne). Die Antwort auf die Frage, ob es ihm etwas ausmachen würde, wenn seine Kinder Deutsche heiraten würden, spiegelt seinen Konflikt deutlich wieder: - por una parte me daria igual + pero por otra no. Wahrscheinlich wäre die Tatsache, daß seine Kinder sich voll in eine Gesellschaft, die inm die Integration verweigert hat, integrieren, schmerzhaft für ihn, denn es würde einem Verrat von Seiten seiner Kinder an ihm gleichkommen oder zumindest einer Niederlage.

Er mag dazu tendieren, alles Spanische überzubewerten, jedoch kann man bei ihm nicht von einer blinden Verehrung Spaniens sprechen. Bei zwei Informanten dieser Gruppe treffen wir sogar eine recht kritische Einstellung gegenüber beiden Ländem an:

Rafael (26): - las papas se cogen aqui igual que en España - los alemanes no son tan cabezas cuadradas como dicen + hay algunos españoles que lo son más todavia. Rafael versichert, daß er nach Spanien zurückgehen würde, wenn er dort die gleiche Arbeit wie in Deutschland hätte.

Die Hinwendung zu ihrer Herkunftsgruppe spiegelt sich im Gebrauch, den diese jungen Leute von der institutionellen Unterstützung der spanischen Sprache und Kultur in Deutschland machen. Drei von ihnen sind Mitglied im spanischen Club, und die restlichen beiden sind es nicht, weil an ihrem Wohnort eine solche Institution nicht existiert oder weil es ihnen aus beruflichen Gründen unmöglich ist. Am meisten fällt bei allen fünf Informanten dieser Gruppe die gute Beherrschung der spanischen Sprache auf, welche wahrscheinlich auf den Besuch einer spanischen Schule in Deutschland (jeweils 10; $2 ; 8$; 5; 0 Jahre), auf die schon erwähnte Mitgliedschaft im spanischen Club, auf die tägliche Umgebung (zwei der Informanten sind mit spanischen Emigranten der zweiten Generation verheiratet, zwei arbeiten in einer spanischen Umgebung und vier von ihnen haben mehr spanische als deutsche Freunde;) und auf ihre eigene positive Einstellung und die ihrer Eltern gegenüber Spanien zurückzuführen ist. Bei vier der Jugendlichen dieser Gruppe wurde dieses Ergebnis durch gute Ergebnisse im Lesetest unterstrichen.

Obwohl die Informanten der zweiten Gruppe die spanische Sprache wesentlich besser beherrschen als die der ersten (die Jugendlichen der ersten Gruppe hatten, von einer Ausnahme abgesehen, alle einen deutschen Akzent im Spanischen; ihr Vokabular ist in dieser Sprache sehr begrenzt, und die syntaktischen Strukturen sind recht arm und fehlerhaft, und vor allem fehlt innen die flüssige Sprachbeherrschung des Muttersprachlers), konnten wir keinen Unterschied bzgl. ihrer Sprachkenntnisse im Deutschen feststellen. In allen Fällen ist das Deutsche die dominante Sprache, in der sich alle am sichersten fühlen. Alle Informanten, ohne jede Ausnahme, beherrschen das Deutsche wie ihre Muttersprache, was der Theorie widerspricht, daß die Hinwendung zur Herkunftsgruppe eine Minderung in der Beherrschung der Sprache der dominanten Gruppe mit sich bringt. Aber es handelt sich bei unseren Informanten, wie wir oben schon betonten, um sehr moderate und relativ kritische Positionen, die gewöhnlich keine extremen Resultate hervorrufen.

\section{Ambiguität und Apathie:}

Die Individuen der beiden letzten Gruppen haben gemeinsam, daß sie sich nicht einer der beiden Gruppen zugewandt haben, sondern beiden. Jedoch unterscheiden sie sich durch ihre Perspektive: Die Mitglieder der ersten Gruppe sehen ihre Zugehörigkeit zu zwei soziolinguistischen Gemeinschaften als eine Verminderung oder gar Aufhebung ihrer Identitäten, während die der zweiten sie als einen wertvollen Zuwachs betrachten.

Die Gruppe der Individuen, die eine zweideutige und apathische Haltung zeigen, besteht aus elf Infor- 
manten (sechs Jungen und fünf Mädchen). Diese jungen Leute haben noch keine klare und befriedigende Identität entwickelt. Sie schwanken ständig zwischen der Zugehörigkeit zu einer der beiden soziolinguistischen Gruppen, ohne sich jedoch für eine von ihnen entschließen und ohne sie beide zusammen in sich vereinigen zu können. Ihr Konflikt ist bewußt, und sie alle leiden darunter.
Ein ähnlich konfliktiver Fall ist der von Angel (28). Sein Konflikt ist ihm jedoch bewußt, und er versucht, inn auf intellektueller Ebene auszutragen. Er selbst stellt seine Situation so dar: - tengo problemas con mi mismo + porque es muy difícil para mi decir + soy español o qué hago + me quedo aqui me quedo alli + siempre estoy payá y pacá (sic) y verdaderamen. te no hago nada + otros chavales de mi edad tienen
Ascención (23) empfindet für die Deutschen nur Verachtung: - a mi no me gusta la manera de ser de los alemanes (...) son antipáticos + a mi los alemanes no me van + no son abiertos + son muy cerrados +10 suyo es lo suyo y lo de uno es de uno.

Sie gibt an, daß sie sich ganz und gar als Spanierin fühlt, sagt aber sofort anschließend: - bueno + totalmente tampoco lo diría + pero (...) dividida + pues entonces igual que yo.

Sie zieht das Spanische auf allen Gebieten vor: - hombre la gente + el ambiente + las cosas tan boni-

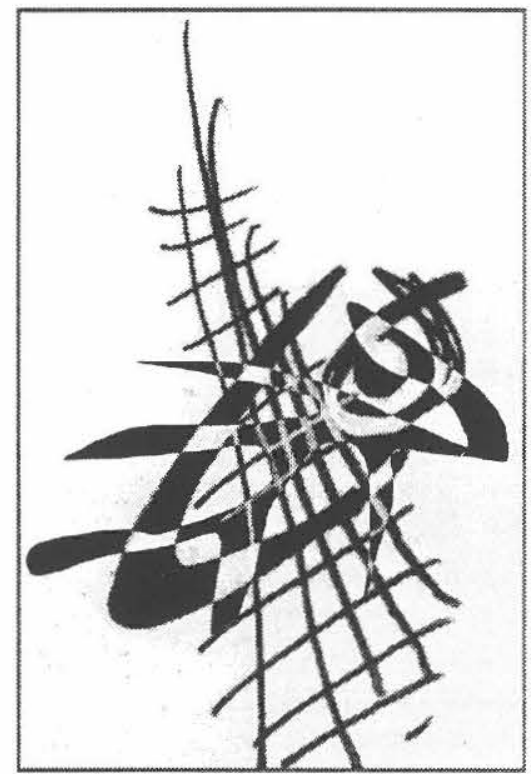
ideas de hacerse una casa + comprarse una casa y establecerse aqui bien + por otro lado tengo ganas de irme a España y luego + por el otro lado también tengo miedo de dejar aqui una existencia ya hecha + tengo mi trabajo tengo mi casa tengo un coche puedo ir de vacaciones $y$ eso es un problema para mi . tengo miedo por dejar la seguridad que tengo aqui + eso es el problema más grande + que estás tan seguro aqui que no te puede pasar nada y tienes miedo de perder tu trabajo + perder tus seguros que tienes + tu paga de renta + tú estás muy bien guar. dado aqui. Anschließend erklärt er, was wahrscheinlich für alle tas que hay. Wir haben hier ein typisches Beispiel für das Phänomen der "myth culture". Ascención weiß genau, daß sie sich schon rein äußerlich von den Deutschen unterscheidet. Auf unsere Frage, ob die Deutschen merken, daß sie keine Deutsche ist, antwortet sie: - claro que si que lo notan (...) se nota en moreno y eso se nota en seguida. Wir vermuten, daß ihre außergewöhnlich dunkle Erscheinung die Ursache für ihre Frustration ist, da sie ihr eine vollständige Integration in die deutsche Gesellschaft unmöglich gemacht hat, und daB ihre Verachtung eine Abwehrreaktion als Folge dieser Erfahrung ist. Wir sehen diese Annahme in den folgenden Antworten bestätigt: - ¿te sientes marginada? - tampoco + me da igual, was natürlich eine völlig widersprüchliche Aussage ist. - trato a los turistas alemanes en España como ellos me tratan a mí. Es ist ihre Art der Rache gegen eine Gesellschaft, die ihr die Aufnahme als gleichwertiges Mitglied versagt.
Informanten dieser Gruppe gültig ist: - la situación ideal para mi seria encontrar una empresa alemana que abre un negocio en España y me manda a Espa. pagando aqui mis derechos + eso sería una situación ideal y a los dos o tres años que yo veo cómo está la vida alli + poder la posibilidad de decir + vale + hemos hecho un contrato de tres años y no me gusta estar aqui + quiero volverme a Alemania y me pagan la vuelta a Alemania (...) la mayoria que se van de aqui se van a lo ciego + el otro se va + dice yo tengo trabajo para medio año + que en España son muy malos + le dan trabajo para medio año y fuera a la calle y asi se van todos y eso es muy dificil cada vez me siento menos alemán + ich distanziere mich nach hier und distanziere mich nach da und steh nachher alleine irgendwo + in der Mitte. Offensichtlich handelt es sich in diesem Falle nicht nur um ein Identitäts-, sondern auch um ein finanzielles Proña a mi + me pagan una paga alemana y me siguen 
blem. Angel ist vollständig in die deutsche Gesellschaft integriert, und er hat sogar einen privilegierten Arbeitsplatz.

Genau wie alle anderen Informanten spricht er Deutsch besser als Spanisch, was er selbst ". muy triste" findet.

Wir beobachteten bei Angel ein sehr interessantes Phänomen: der Sprachenwechsel vom Spanischen ins Deutsche brachte bei inm einen authentischen Charakterwandel mit sich. Die zuvor noch recht schüchterne Person wurde plötzlich verbal impulsiv und hitzig.

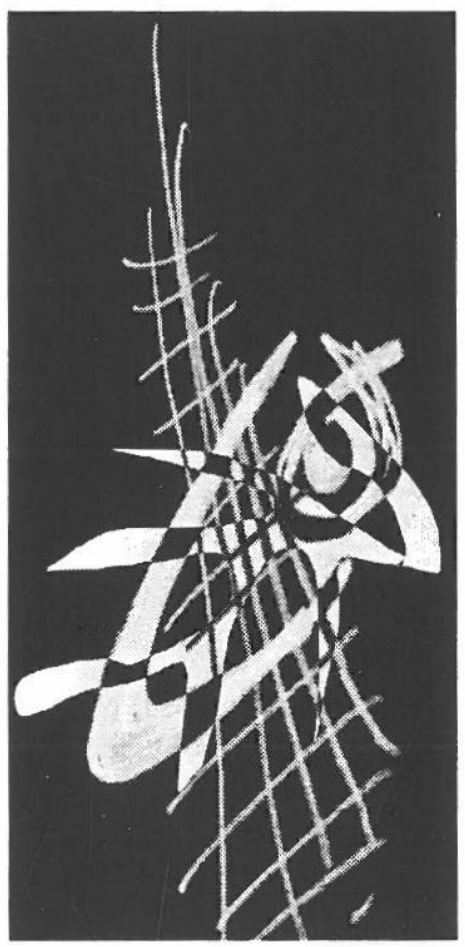

Bei Anita (27) ist der Konflikt weniger offenkundig: - ¡soy española! de verdad + de corazón y todo + pero mi pensamiento mi ser ya no pega (sic) a los españoles - soy española y me moriré española. Es handelt sich um rein sentimentale Aussagen, die jedoch ohne jede Konsequenz bleiben. Anita will unter keinen Umständen nach Spanien zurückgehen, und sie will mit den Deutschen und so wie die Deutschen leben. Sie weiß, daß ihr Spanisch, obschon flüssig und ohne ausländischen Akzent, recht arm und begrenzt ist. Trotzdem ist sie nicht motiviert, dieses Defizit zu beheben. Außerdem kann sie sich lediglich in einer sehr begrenzten Variante des Andalusischen ausdrücken, was natürlich bei anderen Spaniern einige Befremdung hervorruft.

Bei der Leseübung, die ihr sehr schwerfällt, sagt sie: - eso es lo que no me gusta + porque ya esas pala. bras + ya no las entiendo (...) y ya digo + pues no + para eso me leo el periódico alemán y lo entiendo todo.

Würde man von ihrem expliziten Bekenntnis zum Spanischsein absehen, müßte man Anita unserer ersten Gruppe zuordnen, nämlich denen, die die Assimilation an die dominante Gruppe anstreben.
Die Aussage Cristinas (20) weist deutliche Parallelen zu denen Anitas auf: - por el momento me siento un poco más alemana + porque estoy aqui y soy como la gente de aqui ¿no? porque pienso tambièn asi + pero de emoción me siento más española. Sie spricht so, als könne sie ihre Identität in zwei Häiften teilen, eine emotionale und eine rationale. Dies scheint für sie eine machbare Lösung zu sein, wenn auch keine sehr befriedigende, da es sich um eine Teilung und nicht um eine Ergänzung handelt.

Francisco (17) erzählt: - como llego a España + pues me dicen el alemán + como llego aqui a Alemania me dicen el español. Er versichert, daß er sich mehr als Spanier denn als Deutscher sieht. Trotzdem will er nicht nach Spanien zurückgehen, selbst dann nicht, wenn er eine Million Mark hätte. Er sieht sein zukünftiges Leben - casi como un alemán. Er weiß, daß er sich rein äußerlich von den Deutschen unterscheidet, jedoch ist er davon überzeugt, daß er deshalb nicht anders von ihnen behandelt wird.

José (25) beschreibt seine Situation folgendermaßen : - soy español y por la manera de ver soy alemán + pero estoy viviendo en un pais que soy extranjero por el nombre - la manera mia de ser casi es ser alemán y no español + yo me he criado aqui y como aqui hay mucho más alemanes que españoles + por eso. Er erzählt, daß er gelegentlich sogar Schwierigkeiten mit den Spaniern wegen seiner defizitären Ausdrucksweise im Spanischen hatte, sowohl in Spanien als auch in Deutschland, d.h. er ist nicht nur von der spanischen Gesellschaft in Spanien zurückgestoßen worden, sondern auch von der in Deutschland lebenden, der spezifischen Gruppe, der er in ganz besonderem Masse angehört. - estoy contento aqui + pero estaria más contento en España + si tenia (sic) la misma forma de estar aqui. Der Konflikt ist offensichtlich. Die spanische Nationalität ist eine Bürde, die ihm auferlegt wurde, und nicht ein verinnerlichtes Empfinden. Jedoch akzeptiert er diese absurde Situation. Später fügt er hinzu: -cuando estoy en casa soy español + cuando estoy con alemanes + pues me siento más alemán + no pienso en eso.

Als wir ihn nach seiner Erfahrung in Spanien fragten, sagte er: - se nota que no estoy viviendo alli + se nota que también soy extranjero alli - no me tratan 
como uno de los suyos porque yo el idioma español lo hablo como un alemán. Über seine Erfahrungen in der deutschen Schule erzählt er: - cuando iba a la escuela alemana mucha gente se metian conmigo porque era extranjero. Erneut zeigt sich das gleiche tragische Gefühl, ein Ausländer in beiden Ländern zu sein, und dieses Gefühl, keiner der beiden Gruppen als vollständiges Mitglied anzugehören, macht die Identifikation sowohl mit jeder einzelnen als auch mit beiden Gruppen gleichzeitig unmöglich. José ist, wie alle Informanten der dritten Gruppe, ein vaterlandsloses Individuum, das von beiden Gesellschaften, denen er von Rechts wegen angehört, zurückgestoßen wird.

Cristina (20) (Frage unsererseits:) - ¿ha tenido alguna vez problemas a causa del idioma? - en España nada más de la emoción que siempre dicen que viene la alemana. (Frage:) - iha tenido alguna vez problemas por la condición de española, de extran. jera en Alemania? - nada más + a lo mejor + en situaciones de peleas + pues si + te han dicho a lo mejor + dichoso Gastarbeiter o algo - me siento un poco más alemana porque estoy aqui y soy como la gente de aqui + porque pienso también asi + pero de emoción me siento más española. Es ist ganz natürlich, daß sie sich gegenüber der gelegentlichen Ablehnung vonseiten der spanischen und der deutschen Gesellschaft einen Teil ihrer Identität für die Spanier, in diesem Fall den emotionalen und einen Teil für die Deutschen reserviert, nämlich den rationalen Teil. Dadurch bleibt ihr die Möglichkeit, in der einen Gesellschaft Zuflucht zu suchen, wenn sie von der anderen zurückgestoßen wird. Der Konflikt ist ganz offensichtlich, jedoch neigt sich die Waage eher der deutschen Seite zu.

Der Fall von Carmen (22) liegt ähnlich. Auf die Frage, ob sie sich in Spanien unwohl fühle, antwortete sie: - en principio si + porque es raro + porque no sé si soy española o alemana + cuando estoy aqui quiero yo estar alli y cuando pasan tres o cuatro semanas pienso venirme otra vez para Alemania + porque + naja + me gusta + pero + es todo + otra vez con alemanes + estar juntos y entender más alemán y todo eso.

Tino (26) ist ganz sicher, daß er eines Tages nach Spanien zurückkehren wird. Wenn er dort eine Arbeit hätte, würde er sofort gehen. Als wir ihn fragten, ob das Klima allein ein Anlaß für ihn wäre, nach Spanien zurückzugehen, antwortete er: - cuando (sic) era sólo el clima + me podia también ir a algún otro sitio del mundo + sabes + yo he nacido aqui + toda mi familia está en España + nosotros tenemos nadie más aqui + mi madre y mi hermano + yo tengo aqui + he vivido aqui he nacido aqui estaba aqui en la escuela pero mi familia no está aqui (...) no me molesta pero hay algunas veces que me hace falta algo y por eso me quiero también ir algún dia a España + por el tiempo por la familia por todo y la vida española me gusta mejor (...) los alemanes son de una manera un poco fria + lo único que tienen en la cabeza del trabajo y eso + los españoles tienen una otra manera de vivir

Miguel (15) versteht sich besser mit den Türken als mit den Deutschen, obwohl die meisten seiner Freunde Deutsche sind. Er fühlt sich nicht als Außenseiter in Deutschland, hingegen fühlt er sich anfangs, wenn er nach Spanien kommt, recht seltsam. Einerseits erzählt er, daß er eines Tages nach Spanien zurückgehen möchte, und andererseits sieht er seine Zukunft im Mercedeswerk in Kassel. Sein linguistisches Verhalten im Spanischen zeichnet sich durch Unsicherheit aus und durch die Angst, sich lächerlich zu machen.

Die Situation der restlichen drei Informanten ist ähnlich, obwohl sie nicht explizit von ihrem Konflikt spra. chen.

Da wir das Interview der zwei jüngsten Informanten, Blas (9) und Estefania (10), unter der Aufsicht der Eltern machen mußten, sind die Daten nicht sehr zuverlässig, denn beide Eltern sind außergewöhnlich streng und erlauben keinerlei deutschen Einfluß innerhalb ihrer Wohnung. Unter diesen Umständen hätten die Kinder gar nicht zugeben können, daß sie sich als Deutsche sehen, obwohl man anhand ihrer Antworten erkennen kann, daß es in Wahrheit zumindest teilweise so ist, z.B. als wir die beiden Geschwister in einem kuren Moment der Abwesenheit der Eltern auf deutsch fragten, in welcher Sprache sie untereinander sprechen, gaben sie $\mathrm{zu}$, daß sie sich lieber auf deutsch verständigen, wenn ihre Eltern nicht anwesend sind: - manchmal ist mir Deutsch auch etwas einfacher als Spanisch. Aber als wir die

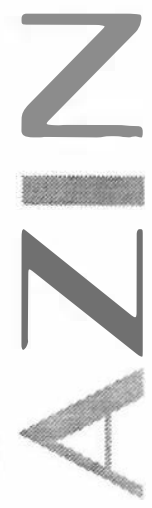


gleiche Frage in Anwesenheit der Eltern auf Spanisch stellten, antworteten sie, daß sie immer Spanisch miteinander sprechen, weil sie diese Sprache besser beherrschen. Indem sie dies sagen, befriedigen sie die Erwartungen ihrer Eltern.

Bezüglich der Diskriminierung vonseiten der Deutschen erzählt Blas, daß einige seiner deutschen Freunde $a b$ und zu im Streit sagen: - que los españoles no valen para nada. Aber er fügt hinzu, daß derjenige, der ihm in diesen Fällen hilft, auch ein Deutscher ist.

In der dritten Gruppe finden wir sowohl Individuen, die sehr gut Spanisch sprechen, als auch solche, die es weniger gut beherrschen. Eine erste Erklärung dafür ist die deutliche Korrelation zwischen der institutionellen Unterstützung des Spanischen am Wohnort und den Sprachkenntnissen der Individuen. Unter den acht Jugendlichen, die in Brühl wohnen, einer Stadt in der es keine Institution gibt, die die spanische Sprache und Kultur pflegt, befinden sich diejenigen, die die schlechtesten Ergebnisse bezügglich der Spanischkenntnisse aufweisen: Mónica, die praktisch einsprachig ist (deutsch) und drei weitere Jugendliche, von denen einer der ersten und zwei der dritten Gruppe angehören. Die Eltern all dieser jungen Leute (drei verschiedene Familien) sehen die besten Zukunftsmöglichkeiten ihrer Kinder in Deutschland und deren völlige Assimilation als unabdingbar auf dem Weg in den sozialen Aufstieg.

Vier der Informanten der dritten Gruppe sprechen das Spanische zwar fließend, jedoch zeichnet es sich durch eine ausgeprägte lexikalische Begrenztheit aus. In zwei Fällen sind die Spanischkenntnisse auf eine stark eingegrenzte dialektale Variante des Andalusischen beschränkt. Die Leseübung brachte in allen Fällen recht defizitäre Ergebnisse. Was jedoch drei von innen von den vier ersten unterscheidet, ist die Einstellung der Eltern, die eines Tages mit ihren Kindern nach Spanien zurückgehen möchten, und denen deshalb die Notwendigkeit bewußt ist, daß ihre Kinder Spanisch sprechen müssen.

Im letzten Fall scheint uns das Alter der Informantin zum Zeitpunkt ihrer Ankunft in Deutschland entscheidend. Es handelt sich um Anita, die mit 6,5 Jahren nach Deutschland kam, in einem Alter, in dem die Grundlagen in ihrer Muttersprache bereits gefestigt waren. Und sie hat diese Sprache nicht verlernt, da sie als Dolmetscherin ihrer Eltern fungieren mußte, sobald sie das Deutsche gerlernt hatte, sogar als Dolmetscherin zwischen ihren Eltern und ihren drei jüngeren Geschwistern, die das Spanische nie ausreichend gelernt haben. Deren Ausdrucksweise im Spanischen klingt wie eine regelrechte Karikatur des Andalusischen, die fast unverständlich ist.

\section{Reaktion der Auflehnung:}

Nur eine Informantin, Aurora (25), kann wegen ihres doppelten und bereichernden Identitätsbewußtseins eindeutig der vierten Gruppe zugeordnet werden. Es ist gleichzeitig die einzige Informantin, die eine Universitätslaufbahn absolviert, und diese privilegierte kulturelle Position ist wahrscheinlich der Hauptauslöser für ihre positive Entwicklung. Sie selbst erklärt ihre Lage so: - me siento bastante alemana + porque la posibilidad que tenemos los chicos que vienen aquí + desde pequeño crecen con dos mentalidades con dos idiomas y dos tradiciones + resulta que tienen la posibilidad de diferenciar lo que es bueno en una nacionalidad lo que es bueno en la otra o lo que es bueno en una mentalidad y en la otra.

Jedoch fühlt sie sich diskriminiert, weil sie als Spanierin in Deutschland kein politisches Wahlrecht hat und laut ihrer Aussage in Zukunft ihren Beruf nicht ausüben darf. - yo reniego a perder una nacionali. dad que siento y coger otra que también siento + yo quisiera tener las dos.

Als wir mit ihr über Basketball sprachen, fragten wir sie, in welcher Mannschaft sie spielen würde, in der spanischen oder in der deutschen. Ihre Antwort gibt in optimaler Weise die Theorie Lamberts wieder: - yo creo que jugaria en la selección europea + yo creo que la terrestre + porque yo soy realmente un ser humano más que nada.

Aurora sucht den Kontakt zu beiden Gemeinschaften, und sie setzt sich mit beiden auf intellektueller Ebene auseinander. Das Resultat ist eine ausgewogene, menschlich gereifte Person, deren Zweisprachigkeit als "koordiniert" bezeichnet werden kann. Die Hypothese Gardners wird hier insofern bestä- 
tigt, als bei Aurora die individuellen soziopsychologischen Faktoren dazu führen, daß sie ihre Zweisprachigkeit als bereichernd erlebt, trotz der soziologischen Umweltfaktoren, die eher eine verarmende Zweisprachigkeit fördern.

Zusammenfassend können wir festhalten, da $\beta$ unsere Untersuchung auf die Gültigkeit unserer Hypothese hindeutet: Die ausschließliche Identifikation mit der dominanten Gruppe führt zu einem diglossischen Prozeß zu Lasten der spanischen Sprache, während die einseitige Identifikation mit der Herkunftsgruppe und/oder die ethnolinguistische Entwurzelung zu einer Situation der Halbsprachigkeit führt. Nur eine doppelte und ausgewogene ethnolinguistische Identität scheint den Erhalt der Herkunftssprache in dieser spezifischen Gruppe aut Dauer garantieren zu können.

\section{K.V.S. $\square$}

\section{BIBLIOGRAPHIE}

- CHILD, I. L. (1943): Italian or American? The Second Generation in Conflict, New Haven: Yale University Press. - GARDNER, R. C. (1982): "Social Factors in Language Retention", in: Lambert, Richard y Barbara Freed (eds.): The Loss of Language Skills, USA, pp. 24-43

- GARDNER, R.N., LALONDE Y J. MAC PHERSON (1985): "Social Factors in Second Language Attrition", in: Language Learning, 35, 4, pp. 519-540.

— GILES, H. UND BYRNE, J.L. (1982): " An Intergroup Approach to Second Language Acquisition", in: Journal of Multicultural and Multilingual Development, 3, pp. 17-40

— GILES, H. UND P. JOHNSON (1981): "The Role of Language in Ethnic Group Relations", in: Turner, J.C. und Giles, H. (eds.): Intergroup Behaviour, Oxford, pp.199-243.

- IDEM (1987): "Ethnolinguistic Identity Theory; a social psychological approach to language maintenance", in: International Journal of the Sociology of Language, 68, pp. 69-99.

- LAMBERT W. E. (1967): "A Social Psychology of bilingualism", in: The Journal of Social Issues, XXIII, 2, pp. 91-109. — IDEM (1977): "The Effects of Bilingualism on the Individual: Cognitive and Sociocultural Consecuences", in: Hornby, P. A.: Bilingualism, Psychological, Social and Educational Implications, pp. 15-27.

- LAMBERT, W.E. , R.C. GARDNER, R. OLTON Y K TUNSTALL (1968): "A Study of the Roles of Attitudes and Motivation in Second Language Learning", in: Fishman, J. A.: Readings in the Sociology of Language, The Hague, pp. 473-491.

— LEETS, L. UND GILES, H.: "Dimensions of Minority Language Survival - Nonsurvival: Intergroup Cognitions and Communication Climates", in: International Journal of the Sociology of Language, (in press).

—SIGUÁN, M. (1976): "Bilingüismo y personalidad", in: Anuario de Psicologia, 15, pp. 275-305

- TOSI, A. (1977): "Semi-Lingualism, Diglossia and Bilingualism; (some observations on the socio-linguistic features of a community of Southern Italians in Britain)" in: Studi, 4, pp. 3-34.

- VILAR SÁNCHEZ, K. (1995a): Lengua y Emigración. Estudio sociolingüístico de los procesos diglósicos entre los jóvenes españoles en Alemania. Granada: Publicaciones de la Cátedra de Historia de la Lengua Española, Universidad de Granada. Idem (1995b): "For Want of the Standard Educated Variety of Spanish ... A German Accent: A sociolinguistic Case Study", in: International Journal of the Sociology of Language (IJSL), 116, p. 5-16.

\section{ANMERKUNGEN:}

'AGER, Asociación Granadina de Emigrantes Retornados, c/Sol y Luna 8, 18007 Granada, Tel.: 958-135333

2 Das Interview bestand aus einem ca.45-minütigen, thematisch geleiteten Gespräch auf Spanisch, an welches sich eine Leseübung und die Nacherzählung einer Bildergeschichte in der gleichen Sprache anschloss. Die letzten 5-10 Minuten sprachen wir mit den Informanten auf Deutsch.

${ }^{3}$ Vilar Sánchez (1995a).

Die akustischen Aufzeichnungen der Interviews können bei der Autorin angefordert werden. Die Anschrift iegt der Redaktion vor.

4 Die linguistische Analyse bestand aus einer exhaustiven Bewertung der Phonetik und Phonologie einerseits und der Morphosyntax ande. rerseits.

${ }^{5}$ Um die Authentizität der Zitate zu gewährleisten, werden die Interpunktionszeichen durch das Pausenzeichen + ersetzt.

${ }^{6}$ In Vilar Sànchez (1995b) findet sich eine ausführliche linguistische Studie des völlig von jeder Norm abweichenden Sprachvernalten Franciscos. 


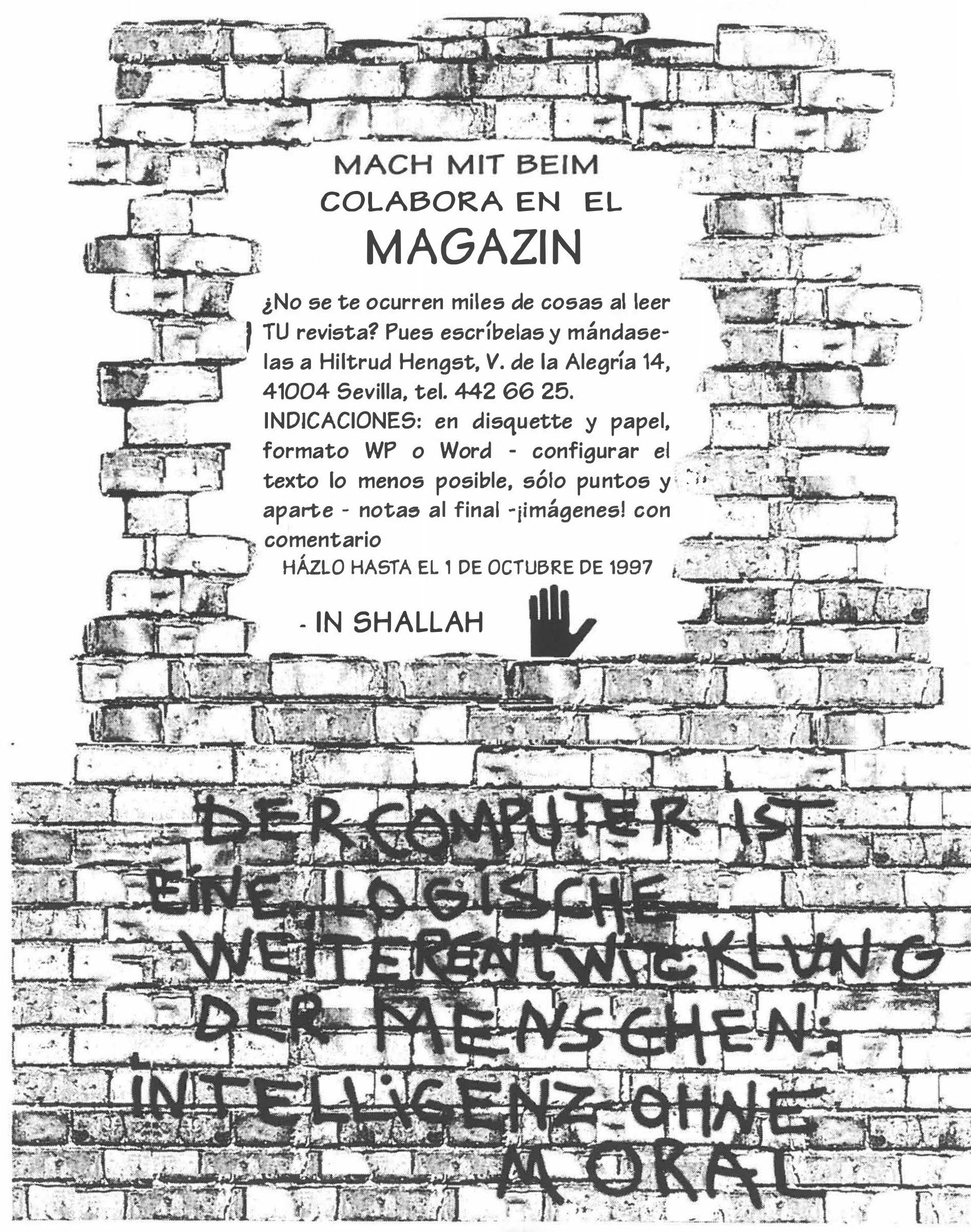

